\title{
Expression and prognostic impact of FZDs in pancreatic adenocarcinoma
}

\author{
Yang Li, Zirong Liu and Yamin Zhang*
}

\begin{abstract}
Background: Despite the high number of researches on pancreatic adenocarcinoma (PAAD) over past decades, little progress had been made due to lack of effective treatment regimens. We aimed to investigate the expression level, mutation, and clinical significance of the Frizzled (FZD) family in PAAD so as to establish a sufficient scientific evidence for clinical decisions and risk management.

Methods: PAAD samples were extracted from The Cancer Genome Atlas (TCGA). Oncomine, Gene expression profiling interactive analysis (GEPIA), human protein atlas (HPA), Kaplan-Meier Plotter, cBioPortal, LinkedOmics, DAVID database, and $\mathrm{R}$ software $(\times 64$ 3.6.2) were used to comprehensively analyze the roles of FZDs. $p$ value below to 0.05 was considered as significant difference.

Results: In total, 179 PAAD tissues and 171 paracancerous tissues were included. The expression levels of FZD1, 2, 6, 7 , and 8 were higher in PAAD tissues than those in normal pancreatic tissue. The higher the expression levels of FZD2 and FZD7, the higher the clinical stage. The overall survival (OS) time was significantly different between low FZD3, 4, 5, 6, and 9 expression group and high expression group. Multivariable analysis showed that FZD3 and FZD6 were independent prognostic factors. The recurrence free survival (RFS) time was significantly different between low FZD4 and FZD8 expression group and high expression group. The RFS difference between low FZD6 expression group and high expression group had not reached statistical significance $(p=0.067)$, which might be due to the small sample size. However, multivariable analysis showed that FZD6 was the only independent factor for RFS. Gene Ontology (GO) and Kyoto Encyclopedia of Genes and Genomes (KEGG) enrichment analysis revealed that FZDs played a critical role in the Wnt signaling pathway, which was further confirmation that FZDs were transmembrane receptors of Wnt signaling pathway.
\end{abstract}

Conclusions: Our results strongly indicated a crucial role of the FZD family in PAAD. FZD3 and FZD6 could be potential prognostic and predictive markers, and FZD6 might also function as a potential therapeutic target in PAAD by blocking Wnt/ß-catenin pathway.

Keywords: Pancreatic adenocarcinoma, TCGA, Fzds, Prognosis

\section{Background}

Pancreatic cancer is one of the most frequently and lethal malignancies of the digestive system with highly aggressive nature [1]. An estimated 57,600 new cases will be diagnosed with pancreatic cancer in the United States

*Correspondence: liyangyizhi@163.com

Department of Hepatobiliary Surgery, Tianjin First Central Hospital, Tianjin 300192, China and 47,050 deaths are projected to occur in 2020 [2]. Pancreatic adenocarcinoma (PAAD) is the most common histological pancreatic cancer subtype, accounting for approximately more than $85 \%$ of all [3]. The current treatment for PAAD is surgery combined with chemo and radiotherapies [4]. Despite the high number of researches on PAAD over past decades, little progress had been made due to lack of effective treatment regimens $[5,6]$. Therefore, a search for new specific 
molecular biomarkers for diagnosis and prognosis of PAAD is urgently needed, which may help in developing targeted diagnostic and therapeutic strategies.

The Frizzled (FZD) family is transmembrane receptors of Wnt signaling pathway, consisting of 10 isoforms (FZD1-10) [7, 8]. FZDs are reported to be involved not only in embryogenesis and development but also in tumor development and progression [9, 10]. FZD2 was over-expressed in hepatocellular carcinoma (HCC) and head and neck squamous cell carcinoma compared to paracancerous tissues and significantly associated with the survival of patients [11]. In endometrial cancer, FZD2 could modulate the EMT process by activating Wnt signaling pathway [11]. Lei Chen et al. [12] reported that miR-101 acted directly on FZD4 to influence the migration and invasion of bladder cancer cells. Downregulation of FZD5 was observed in $\mathrm{CD} 30^{+}$diffuse large B-cell lymphoma (DLBCL) [13]. The dysregulated expression levels of FZD7 was reported in breast cancer, colon cancer and HCC [14], thus promoting tumor proliferation, invasion, and metastasis. However, few studies have been performed on diagnostic and prognostic significance of the FZD family in PAAD. Fakhar et al. [15] showed that Klotho, an anti-aging protein, might suppress the WNT signaling by binding to WNT-1 and cystein-rich domains (CRDs) of FZD-1/2 in pancreatic cancer. Steinhart et al. [16] discovered that antagonistic FZD5 and FZD8 antibody could inhibit the proliferation and growth of PADC cells both in vivo and in vitro. To the best of our knowledge, there are presently no studies assessing the role of the FZD family in PAAD systematically using bioinformatics approach. Herein, we aimed to investigate the expression level, mutation, and clinical significance of the FZD family in PAAD so as to establish a sufficient scientific evidence for clinical decisions and risk management.

\section{Methods}

\section{Oncomine}

Oncomine database is a cancer microarray database, including 715 datasets and 86733 samples (www.oncom ine.org). The expression status of FZDs were investigated using the Oncomine database. Analysis type was set as cancer vs. normal analysis. The threshold of $p$ value and fold change (FC) was set as $<0.05,>1.5$, respectively.

\section{Gene expression profiling interactive analysis (GEPIA)}

GEPIA is a multidimensional cancer genomics dataset which integrated mass data from The Cancer Genome Atlas (TCGA) and the Genotype-Tissue Expression project (GTEx) (http://gepia.cancer-pku.cn/). GEPIA was used to evaluate the gene expression differences between PAAD and normal tissues based on the analysis of variance (ANOVA) and produce the scatter diagram and box plot. The correlation between FZDs and clinical stage was also assessed using GEPIA, and the statistical method used was Pearson correlation coefficient.

\section{Human protein atlas (HPA)}

Images of immunohistochemistry staining for PAAD and normal tissues were collected HPA (https://www.prote inatlas.org/). HPA applied transcriptome and proteomics to provide different protein atlases, including tissue atlas, cell atlas, and pathology atlas.

\section{Kaplan-Meier plotter}

Kaplan-Meier plotter was available to estimate the effect of various genes on prognosis in different cancer types (http://kmplot.com). The PAAD samples were divided into two groups according to the level of expression of FZDs. Overall survival (OS) was considered the time to death or the last follow-up time from the initial diagnosis of PAAD, whereas recurrence free survival (RFS) was the time to relapse from the diagnosis. The hazard ratio (HR) and $p$ value had been labeled.

\section{Univariable and multivariable risk analysis}

All the FZDs that had prognostic impact (OS or RFS) were screened as candidates for univariable and multivariable Cox proportional-hazard regression analysis. HRs and 95\% confidence interval (CI) were calculated for each factor. SPSS software (IBM SPSS 23.0 for Windows, SPSS Inc., Chicago, IL, USA) was used for statistical analysis.

\section{cBioPortal}

cBioPortal, an intuitive Web interface, was applied to perform gene variation analysis of PAAD (http://www. cbioportal.org/), including amplification, mutation, and copy number variation. An overview of genetic alteration of each FZD family member was also provided to visualize complete details of each type of mutation in each individual sample.

\section{Correlation analyses}

Correlation between every two FZDs was assessed using a Pearson's correlation coefficient. Statistical analysis and the graph were finished with $\mathrm{R}$ software $(\times 64$ 3.6.2). $p$ value below to 0.05 was considered as significant correlations.

\section{LinkedOmics database}

Using the online LinkedOmics database, we screened the most relevant genes of each FZD family member. The top 50 genes significantly associated with FZDs were provided in a heat map and volcano plot. 


\section{Gene ontology and Kyoto encyclopedia of genes and genomes}

Gene Ontology (GO) and Kyoto Encyclopedia of Genes and Genomes (KEGG) enrichment analysis were performed in the DAVID database (https://david.ncifc rf.gov/). GO term consisted of three categories: biological processes (BP), cellular component (CC), molecular function (MF). Significant pathway computing was provided in DAVID. The graph of GO and KEGG analysis was plotted by $\mathrm{R}$ packages named ggplot 2 in the $\mathrm{R}$ software $(\times 643.6 .2)$.

\section{Results}

mRNA expression levels of FZDs in human cancers

The mRNA expression levels of each isoform in the FZD family between cancer and paracancerous tissues were determined using the Oncomine database (Fig. 1). In 419 total unique analyses, 45 analyses showed that the mRNA expression level of FZD1 was significantly different between cancer tissue and paracancerous tissue, consisting of 20 up-regulated expression and 25 down-regulated expression. FZD2 was found to be up-regulated significantly in 41 analyses while down-regulated in 4 analyses. FZD3, 6, 7 and 10 also had similar results that their mRNA expression levels were higher compared to paracancerous tissues in more unique analyses. Reduced expression of FZD4 was observed in 40 analyses while increased expression was in 7 analyses. FZD5 8 and 9 followed a similar expression pattern.

\section{mRNA and protein expression levels of FZDs in PAAD}

The GEPIA database was used to determine the mRNA expression levels of FZDs in PAAD (Fig. 2a, b). 179 PAAD tissues and 171 paracancerous tissues were included. Compared to paracancerous tissues, the expression levels of FZD1, 2, 6, 7 and 8 were significantly elevated $(p<0.05)$. There was no significant difference in expression of the other FZD family members (FZD3, 4, 5, 9, and $10)$.

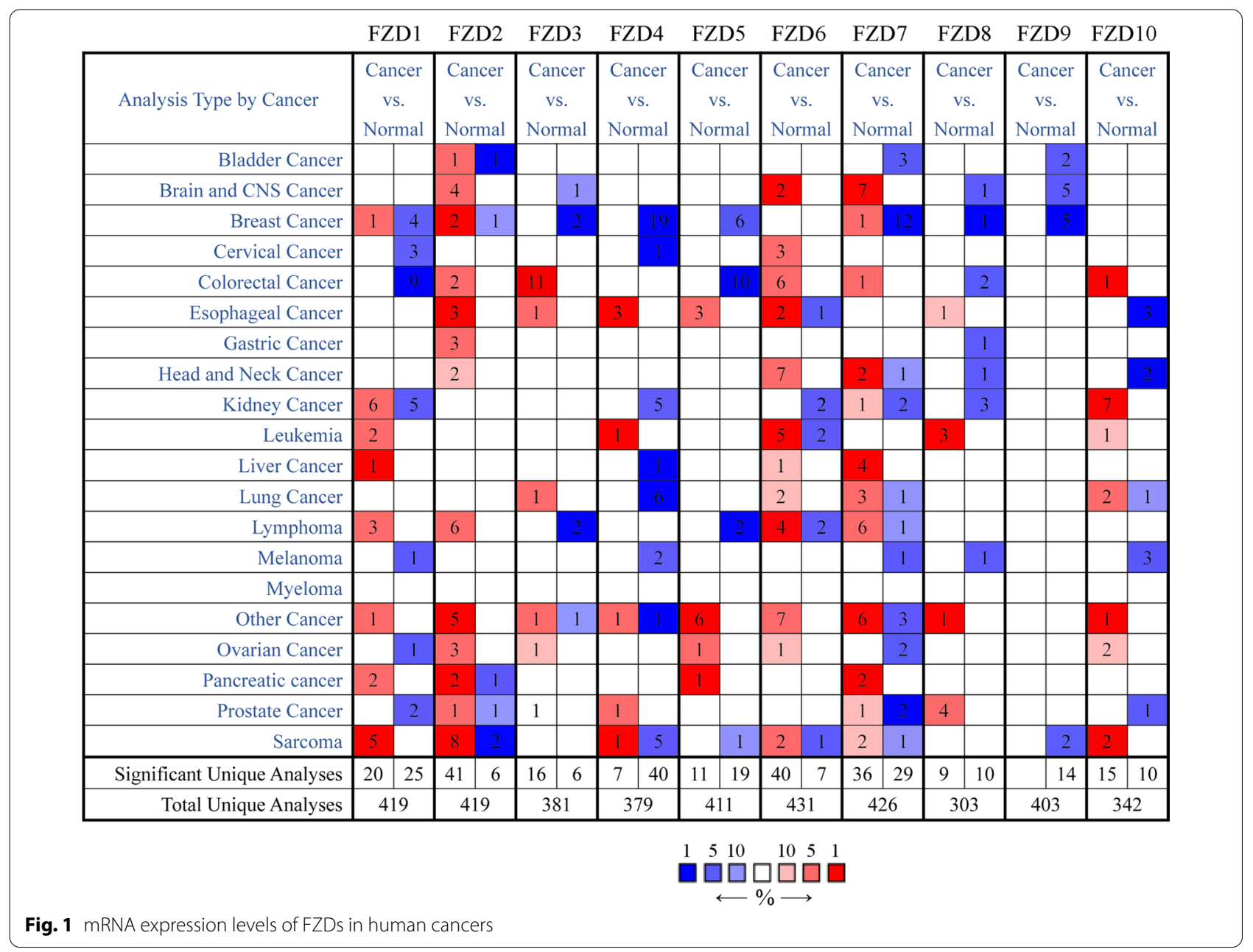




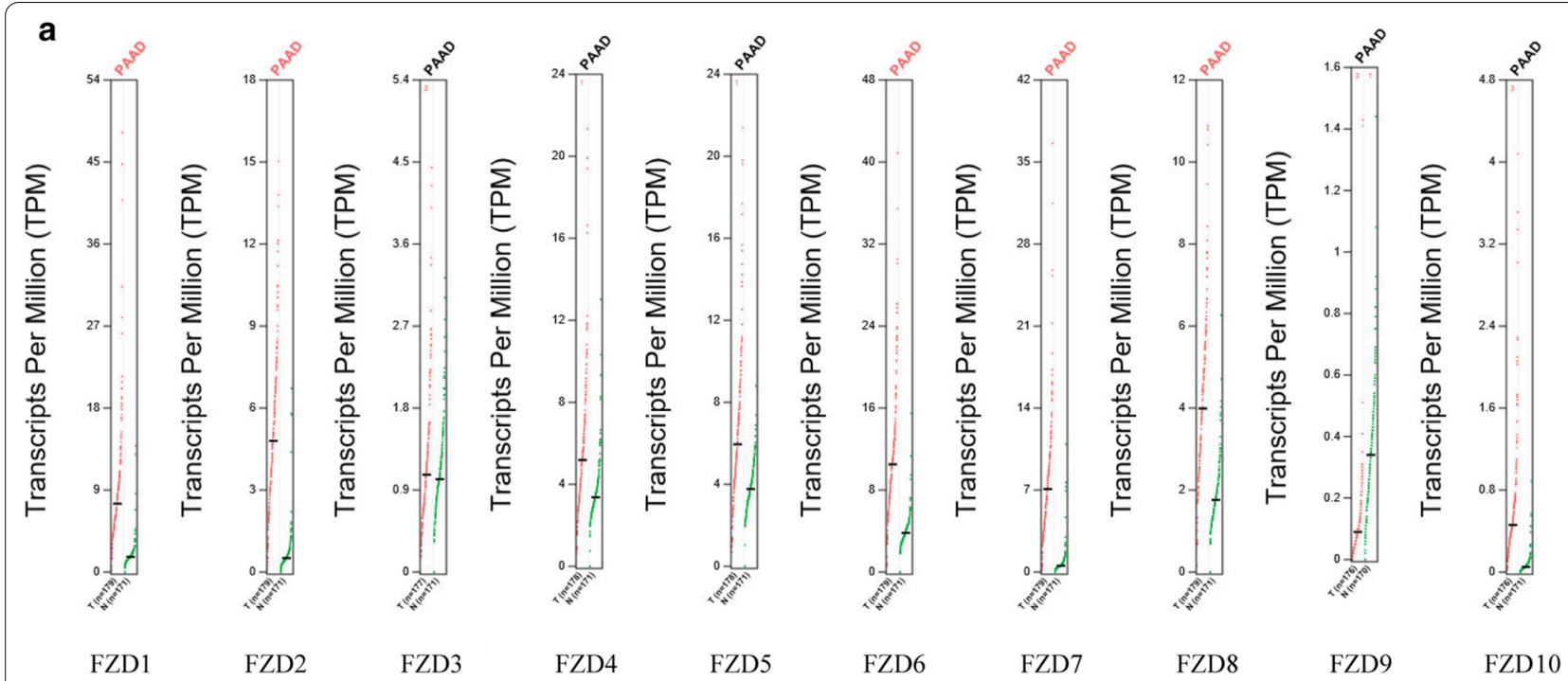

b

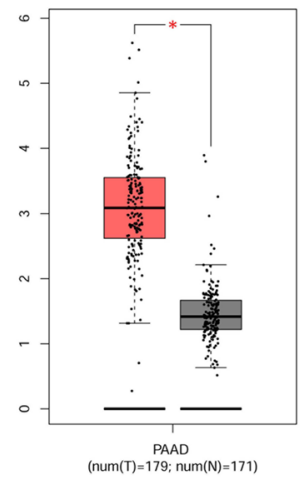

FZD1

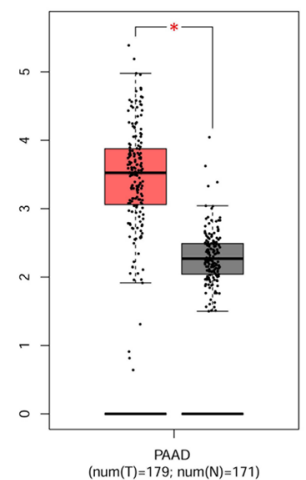

FZD6

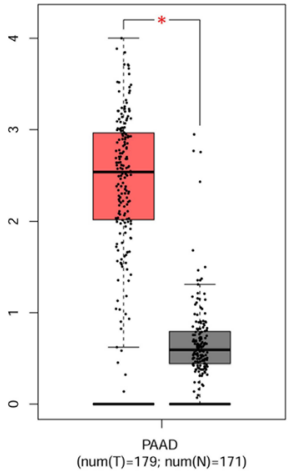

FZD2

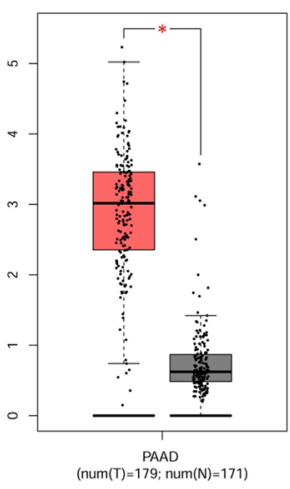

FZD7

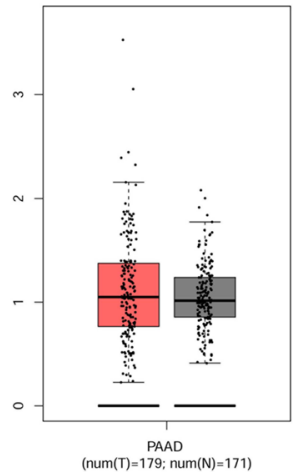

FZD3

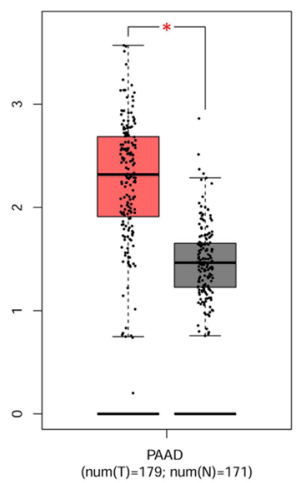

FZD8

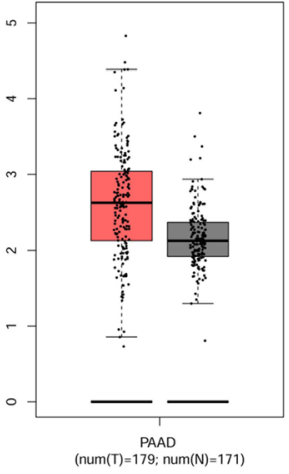

FZD4

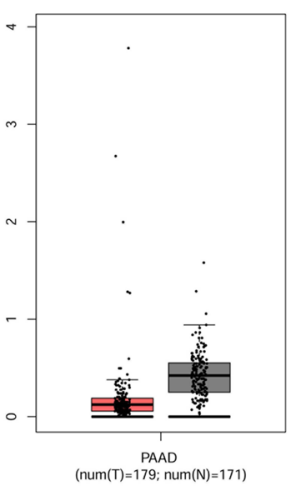

FZD9

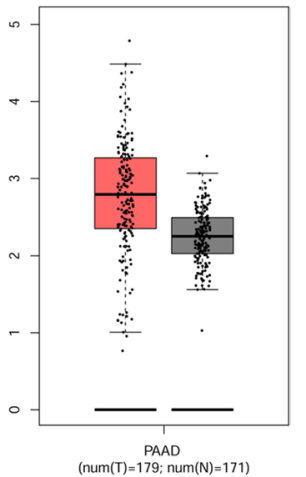

FZD5

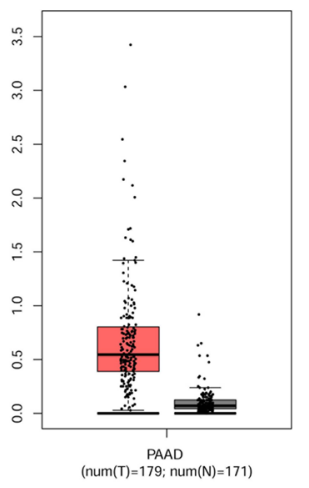

FZD10

Fig. 2 mRNA expression levels of FZDs in PAAD. a Scatter diagram. b Box plot

The protein levels of FZDs in PAAD was studied using the Human Protein Atlas (HPA) (Fig. 3). Aside from missing information for FZD9, the protein levels of FZD1, 2, $3,4,5,6,7,8$, and 10 was increased in PAAD tissues compared with paracancerous tissues.
Relationship between clinical characteristics and FZDs

The relationship between tumor stage and FZDs were examined using the GEPIA database (Fig. 4a). A total of 174 PAAD patients had definite clinical stages, including 20 patients with stage I, 145 with stage II, 4 with stage III, 


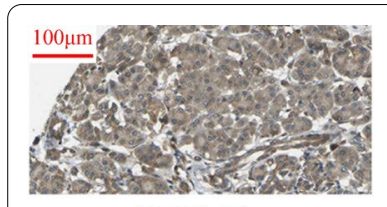

FZD1 (T)

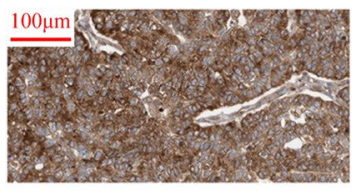

FZD3 (T)

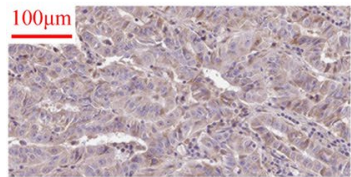

FZD5 (T)

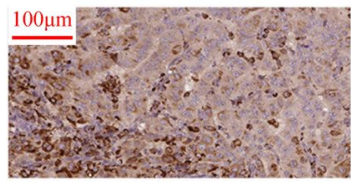

FZD7 (T)

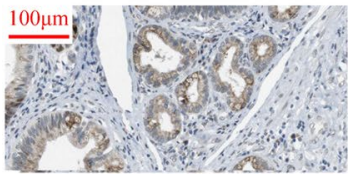

FZD10 (T)

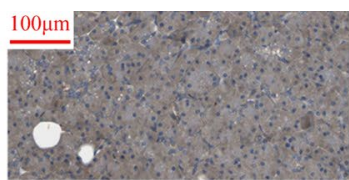

FZD1 (N)

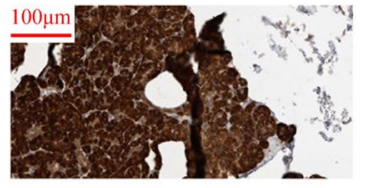

FZD3 (N)

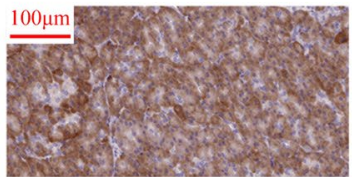

FZD5 (N)

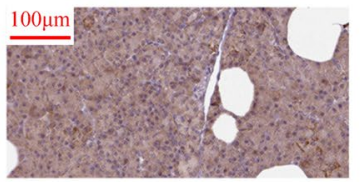

FZD7 (N)

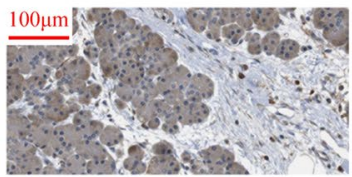

FZD10 (N)

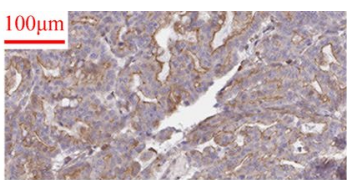

FZD2 (T)

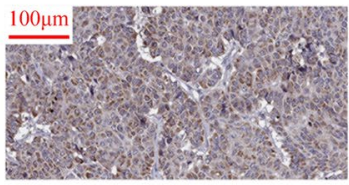

FZD4 (T)

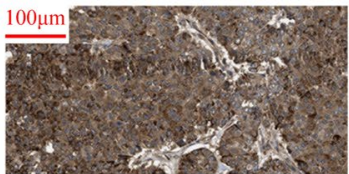

FZD6 (T)

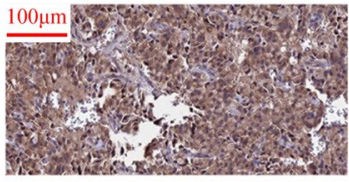

FZD8 (T)

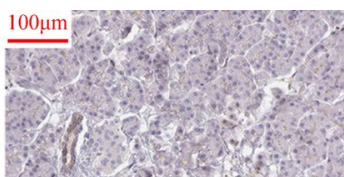

FZD2 (N)

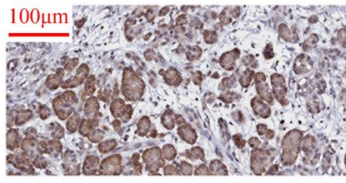

FZD4 (N)

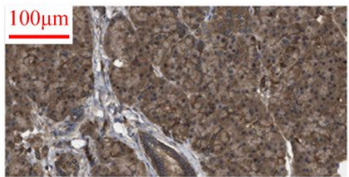

FZD6 (N)

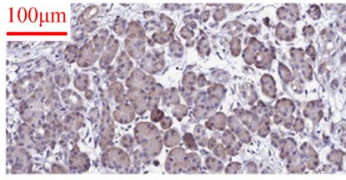

FZD8 (N)

Fig. 3 Protein expression levels of FZDs in PAAD

and 5 with stage IV. The results revealed significant positive correlation of FZD2 and advanced stage $(p<0.01)$. Significant correlation between FZD7 and advanced stage were also observed $(p<0.01)$. FZD5 correlated almost statistically significantly with advanced stage $(p=0.058)$. No significant correlations were observed between other FZDs and tumor stage.

In addition to tumor stage, we also evaluated the effect of FZDs on the prognosis for PAAD using the KaplanMeier Plotter. As shown in the Fig. 4b, patients with FZD3 high-expression group had better OS than the low-expression group $(p<0.05)$ whereas there was no significant correlation between FZD3 expression and RFS. FZD4 high-expression group was significantly associated with better OS and RFS $(p<0.01)$. Contrary to FZD3, FZD5 and 6 high-expression group had worse OS than the low-expression group $(p<0.05)$. The RFS difference between low FZD6 expression group and high expression group had not reached statistical significance $(p=0.067)$, which might be due to the small sample size. For FZD8, patients with the low-expression group had a markedly more favorable RFS $(p<0.05)$ while no significant OS difference was noted between the low-expression group and the high-expression group. Although there is no significant difference in mRNA expression level of FZD9 between PAAD tissues and paracancerous tissues, patients with FZD9 high-expression group had better OS than the low-expression group $(p<0.05)$. The expression level of FZD1, 2, 7, and 10 did not significantly affect the prognosis (OS or RFS) for PAAD.

Further univariable analysis of OS in patients with PAAD showed that age, FZD3 (low-expression group vs. high-expression group), FZD6 (low-expression group vs. high-expression group), and FZD8 (low-expression group vs. high-expression group) were prognostic factors (Table 1). Univariable analysis of RFS showed that FZD6 (low-expression group vs. high-expression group) was the only prognostic factor (Table 1). Multivariable analysis of OS in patients with PAAD showed that older age, low expression of FZD3, and high expression of FZD6 
Li et al. BMC Gastroenterol ～(2021) 21:79

Page 6 of 14

a

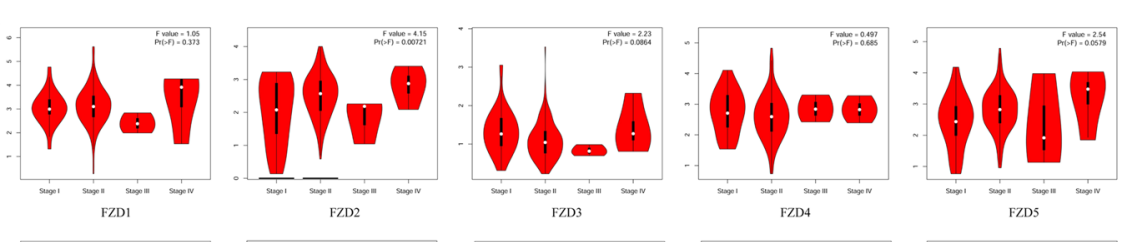

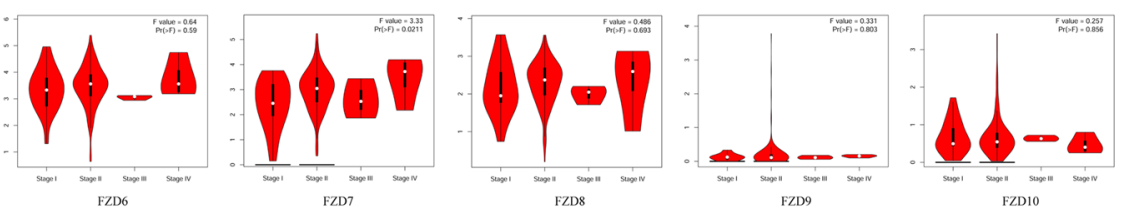

b
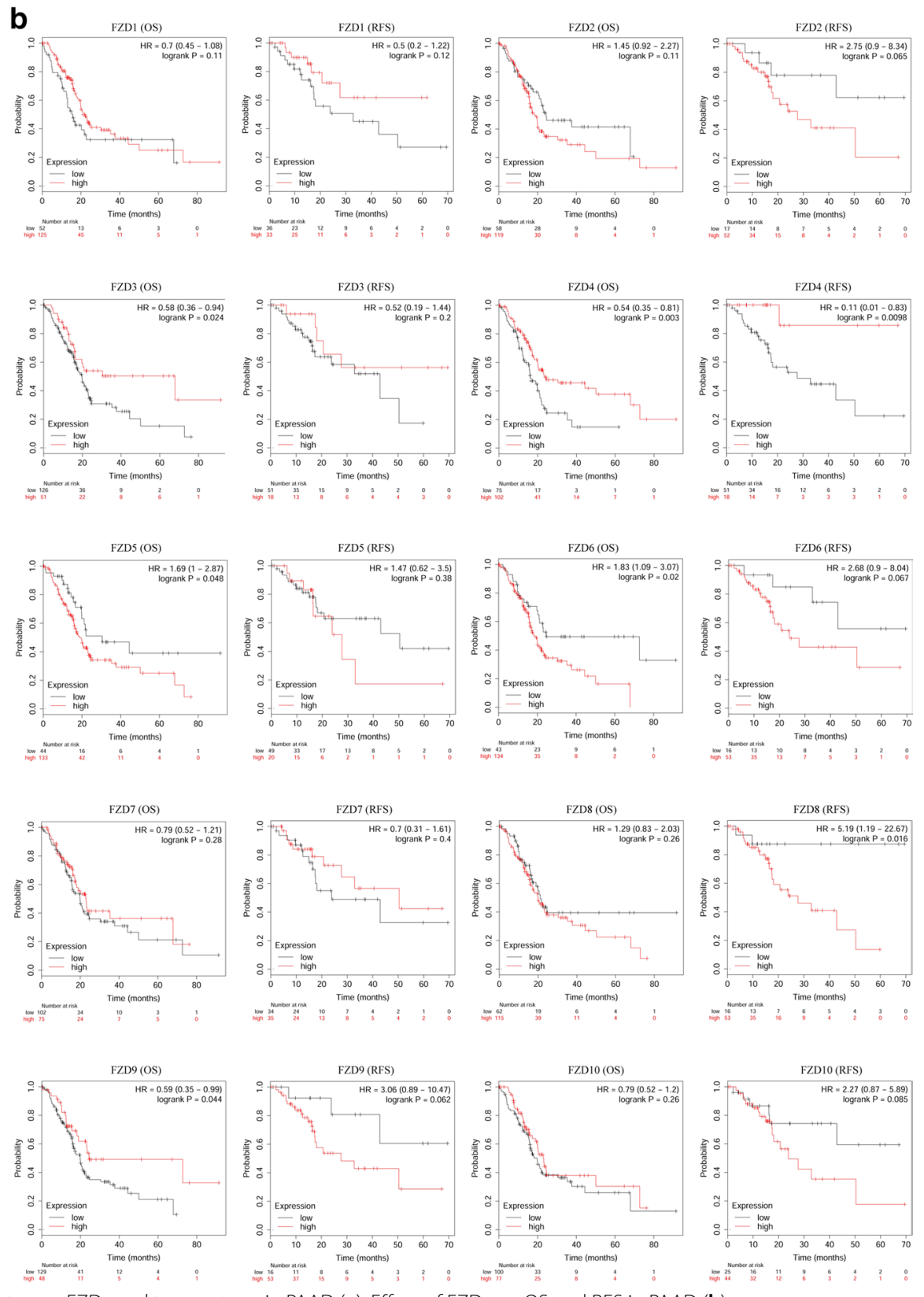

Fig. 4 Correlation between FZDs and tumor stage in PAAD (a). Effect of FZDs on OS and RFS in PAAD (b) 
Table 1 Univariable and multivariable analysis of OS and RFS in patients with PAAD

\begin{tabular}{|c|c|c|c|c|c|c|c|c|}
\hline \multirow[t]{3}{*}{ Variables } & \multicolumn{4}{|c|}{ Univariable analysis } & \multicolumn{4}{|c|}{ Multivariable analysis } \\
\hline & \multicolumn{2}{|l|}{ os } & \multicolumn{2}{|l|}{ RFS } & \multicolumn{2}{|l|}{ os } & \multicolumn{2}{|l|}{ RFS } \\
\hline & $\mathrm{HR}(95 \% \mathrm{Cl})$ & $p$ value & $\mathrm{HR}(95 \% \mathrm{Cl})$ & $p$ value & $\mathrm{HR}(95 \% \mathrm{Cl})$ & $p$ value & $\mathrm{HR}(95 \% \mathrm{Cl})$ & $p$ value \\
\hline Age, years & $1.027(1.006-1.049)$ & 0.012 & $1.024(0.974-1.077)$ & 0.355 & $1.028(1.005-1.051)$ & 0.018 & $1.027(0.964-1.095)$ & 0.409 \\
\hline \multicolumn{9}{|l|}{ Gender } \\
\hline Male & Reference & & Reference & & Reference & & Reference & \\
\hline Female & $1.159(0.768-1.750)$ & 0.483 & $2.199(0.924-5.236)$ & 0.075 & 1.134 (0.729-1.764) & 0.578 & $2.661(0.916-7.729)$ & 0.072 \\
\hline \multicolumn{9}{|l|}{ Grade } \\
\hline $1+\|$ & Reference & & Reference & & Reference & & Reference & \\
\hline$I I I+I V$ & $1.512(0.979-2.335)$ & 0.062 & $1.367(0.561-3.331)$ & 0.491 & 1.199 (0.754-1.908) & 0.443 & $1.278(0.437-3.739)$ & 0.654 \\
\hline \multicolumn{9}{|l|}{ FZD3 } \\
\hline Low & Reference & & Reference & & Reference & & Reference & \\
\hline High & $0.647(0.427-0.983)$ & 0.041 & $0.705(0.301-1.651)$ & 0.421 & $0.639(0.408-1.000)$ & 0.048 & $0.323(0.097-1.072)$ & 0.065 \\
\hline \multicolumn{9}{|l|}{ FZD4 } \\
\hline Low & Reference & & Reference & & Reference & & Reference & \\
\hline High & $0.783(0.518-1.185)$ & 0.248 & $1.416(0.609-3.289)$ & 0.419 & $0.899(0.568-1.422)$ & 0.649 & $1.961(0.680-5.657)$ & 0.213 \\
\hline \multicolumn{9}{|l|}{ FZD5 } \\
\hline Low & Reference & & Reference & & Reference & & Reference & \\
\hline High & $1.001(0.661-1.516)$ & 0.996 & $2.061(0.861-4.931)$ & 0.104 & $0.886(0.572-1.373)$ & 0.59 & 1.107 (0.399-3.072) & 0.845 \\
\hline \multicolumn{9}{|l|}{ FZD6 } \\
\hline Low & Reference & & Reference & & Reference & & Reference & \\
\hline High & $1.680(1.101-2.564)$ & 0.016 & $6.159(2.082-18.214)$ & 0.001 & 1.951 (1.234-3.083) & 0.004 & $9.299(2.510-34.450)$ & 0.001 \\
\hline \multicolumn{9}{|l|}{ FZD8 } \\
\hline Low & Reference & & Reference & & Reference & & Reference & \\
\hline High & $0.600(0.394-0.915)$ & 0.018 & $1.257(0.535-2.956)$ & 0.599 & $0.659(0.414-1.048)$ & 0.078 & $1.634(0.582-4.589)$ & 0.351 \\
\hline \multicolumn{9}{|l|}{ FZD9 } \\
\hline Low & Reference & & Reference & & Reference & & Reference & \\
\hline High & $0.764(0.503-1.161)$ & 0.207 & $0.555(0.235-1.313)$ & 0.180 & $0.878(0.569-1.355)$ & 0.556 & $0.704(0.239-2.075)$ & 0.525 \\
\hline
\end{tabular}

were significantly associated with a greater risk of death (Table 1). Multivariable analysis of RFS showed that high expression of FZD6 was significantly associated with an increased risk of recurrence (Table $1, p=0.001$ ).

\section{Mutation and correlation analysis of FZDs}

The cBioPortal database was utilized to investigate the mutational landscape of the FZD family. 21\% (31/149) patients had genetic alternations and the amplification was most frequent mutation among FZD isoforms (Fig. 5a). More specifically, mutations were most frequently identified for FZD6 (7\%), all of which were amplification mutations (Fig. 5c). Genetic alternations of FZD1, 2 , and 8 included amplification and missense mutations. FZD3 included amplification, missense and deep deletion. FZD4 and 10 only included missense mutations. FZD5 and 7 only included amplification mutations. FZD9 included both amplification and truncating mutations.

Next, we examined the correlation among the FZD members using the Pearson correlation analysis. As shown in Fig. 5b, significantly positive correlations were observed between FZD1 and 2, 4, 6, 7, 8; FZD2 and 6, 7, 8; FZD3 and 6; FZD4 and 7, 8; FZD5 and 8, 9; FZD6 and 7; FZD7 and 8. Significantly negative correlation was observed between FZD2 and 3 .

\section{Correlated significant genes with FZDs}

The LinkedOmics database was used to study the correlated significant genes with the FZD members. The top 50 correlated genes were shown in the volcano (Fig. 6) and heatmap plot (Fig. 7). We found that the most negatively correlated genes with FZD1 included TSTD1, ICA1, and ACP1 while the positively correlated genes with FZD1included DCHS1, GLT8D2, and ZNF521. The most negatively correlated genes with FZD2 included RALGAPA1, GRSF1, and TMED8 while the positively correlated genes with FZD2 included CNN2, PODNL1, and HOMER3. The most negatively correlated genes with FZD3 included TMEM44, TSPO, and ARPC1B while the positively correlated genes with FZD3 included FBXO16, 


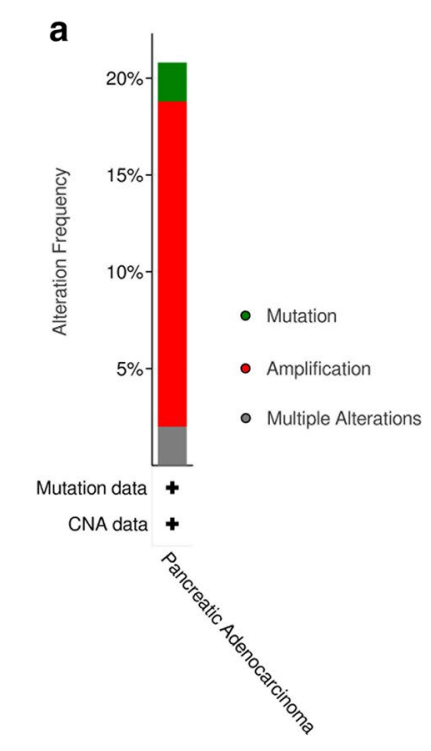

C

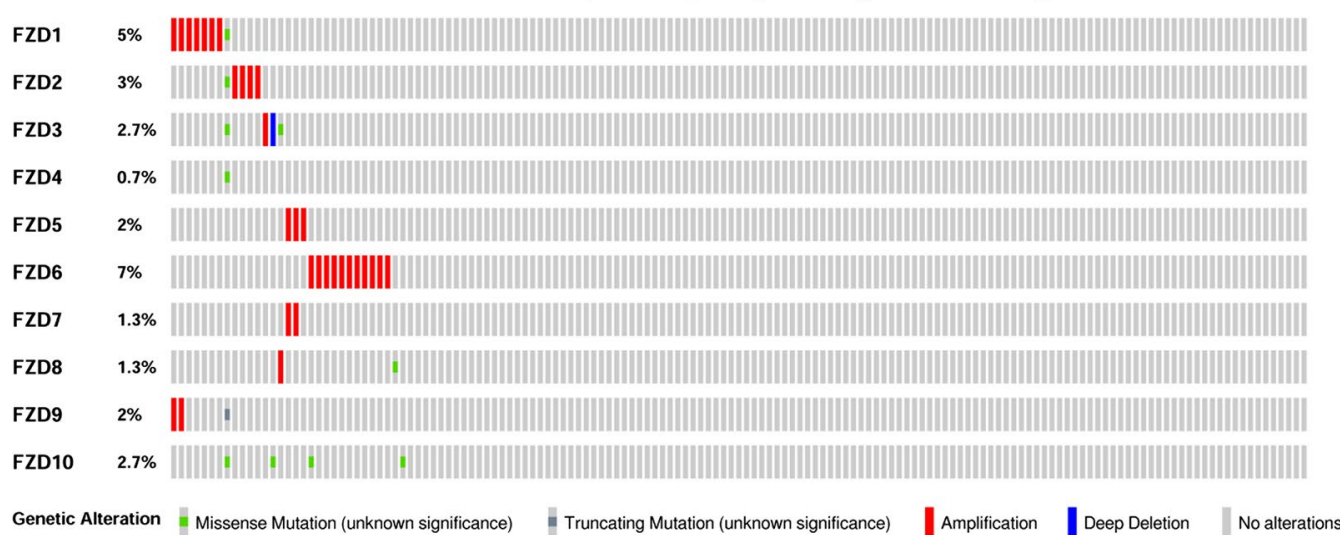

Fig. 5 Mutation and correlation analysis of FZDs in PAAD. a Mutation frequency of FZDs. b correlation between every two FZDs. C Mutation details of every FZD family member in each individual sample

ZFYVE9, and IRAK1BP1. The most negatively correlated genes with FZD4 included ABHD11, ALDOA, and GSS while the positively correlated genes with FZD4 included SHE, ERG, and AYYR1. The most negatively correlated genes with FZD5 included MYL6B, ST3GAL3, and STMN3 while the positively correlated genes with FZD5 included OCLN, POF1B, and CGN. The most negatively correlated genes with FZD6 included REPIN1, HIGD2A, and CST3 while the positively correlated genes with FZD6 included FNDC3B, ADAM17, and PDP1. The most negatively correlated genes with FZD7 included ERP29, $M M A B$, and PEMT while the positively correlated genes with FZD7 included RAB31, FIBIN, and LOXL3. The most negatively correlated genes with FZD8 included EIF2AK1, GRSF1, and CSE1L while the positively correlated genes with FZD8 included CYS1, CCDC8, and ADCY5. The most negatively correlated genes with
FZD9 included FAM126B, LOC220930, and ZMYM5 while the positively correlated genes with FZD9 included IGFALS, IDH2, and SOD3. The most negatively correlated genes with FZD10 included GPKOW, C1orf97, and ISCA2 while the positively correlated genes with FZD10 included ADAMTS4, RNF122, and GPR4.

\section{Functional analysis of FZDs}

FZDs and the above correlated genes (70 genes in total) were subject to GO and KEGG enrichment analysis in the DAVID database. Top five processes were shown in Fig. 8a-c. Notably, there were four BP processes associated with Wnt signaling pathway (GO: 0060071, 0060070 , 0035567, 007223) and two MF processes associated with Wnt signaling pathway (GO: 0042813, 001714). These indicated that FZDs were very relevant to Wnt signaling pathway. As KEGG analysis showed, Wnt signaling 

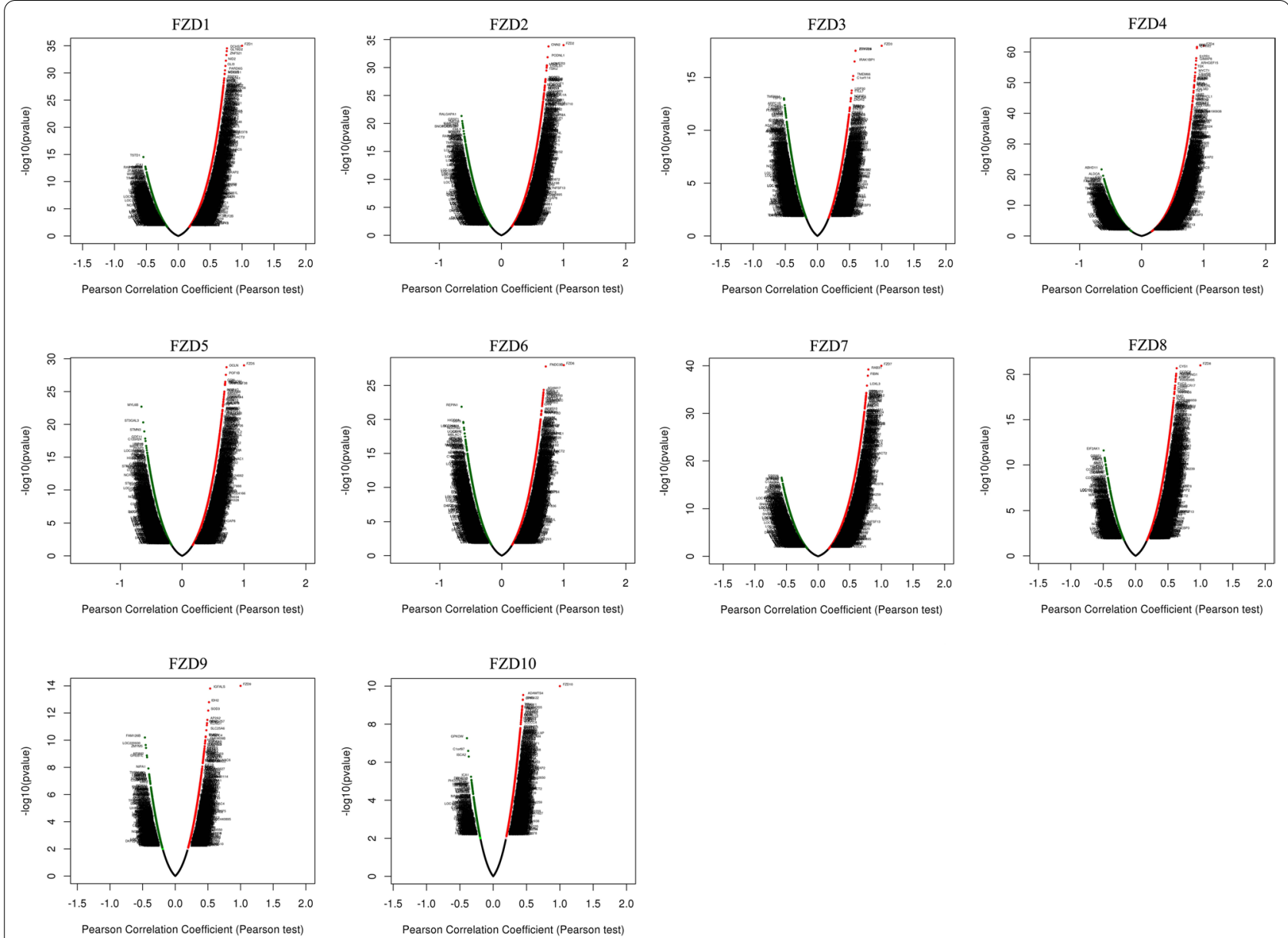

Fig. 6 Volcano plot of top 50 correlated genes to FZDs

pathway was one of the top 5 enrichment pathways (Fig. 8d). Wnt proteins took part in adherens junction, cell sycle, proteolysis, cytoskeletal change, gene transcription and DNA processes by acting on FZDs (Fig. 9).

\section{Discussion}

Using different public databases, we have provided a comprehensive analysis of FZDs in PAAD patients for the first time. The expression levels of FZD1, 2, 6, 7, and 8 were higher in PAAD tissues than those in normal pancreatic tissue. The higher the expression levels of FZD2 and FZD7, the higher the clinical stage. PAAD patients with high expression of FZD6 had shorter OS than those with low expression, indicating that FZD6 could be a potential prognostic and predictive marker in PAAD. PAAD patients with high expression of FZD8 had shorter RFS than those with low expression, indicating that FZD8 could be a potential therapeutic target in PAAD. GO and KEGG enrichment analysis revealed that FZDs played a critical role in the Wnt signaling pathway, which was further confirmation that FZDs were transmembrane receptors of Wnt signaling pathway.

FZD1 has been reported to be biological markers for PAAD [17]. They indicated that positive FZD1 expressions were closely with poor prognosis in a retrospective study including 106 PAAD patients. Their observations were not consistent with our results. Our study collected 176 cases and no significant survival differences were observed between high expression group and low expression group $(p=0.11)$. However, the expression level of FZD1 increased significantly and could be alternative target for the treatment of PAAD. This was also evidenced by a study form Bharti et al. that triptolide could inhibit the tumor growth of pancreatic cancer by inactivate WNT1 and FZD1 [18].

FZD2 functioned as an oncogene in various cancers. FZD2 shRNA could suppress the proliferation, migration, and invasion of gastric cancer cells [19]. In endometrial cancer, elevated FZD2 promoted the migration 

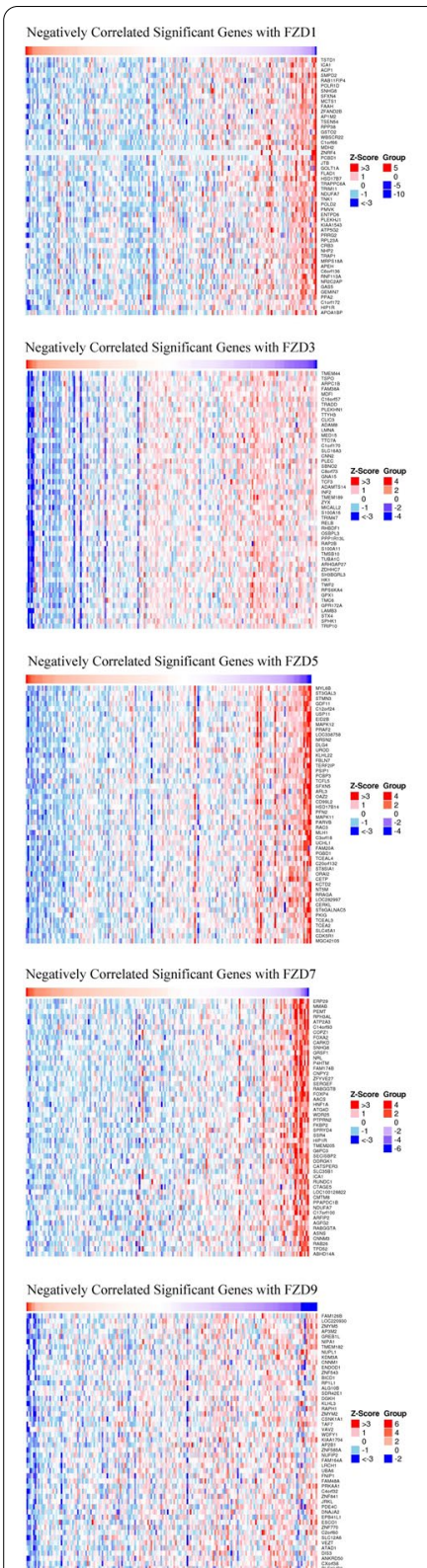

Fig. 7 Heatmap plot of top 50 correlated genes to FZDs
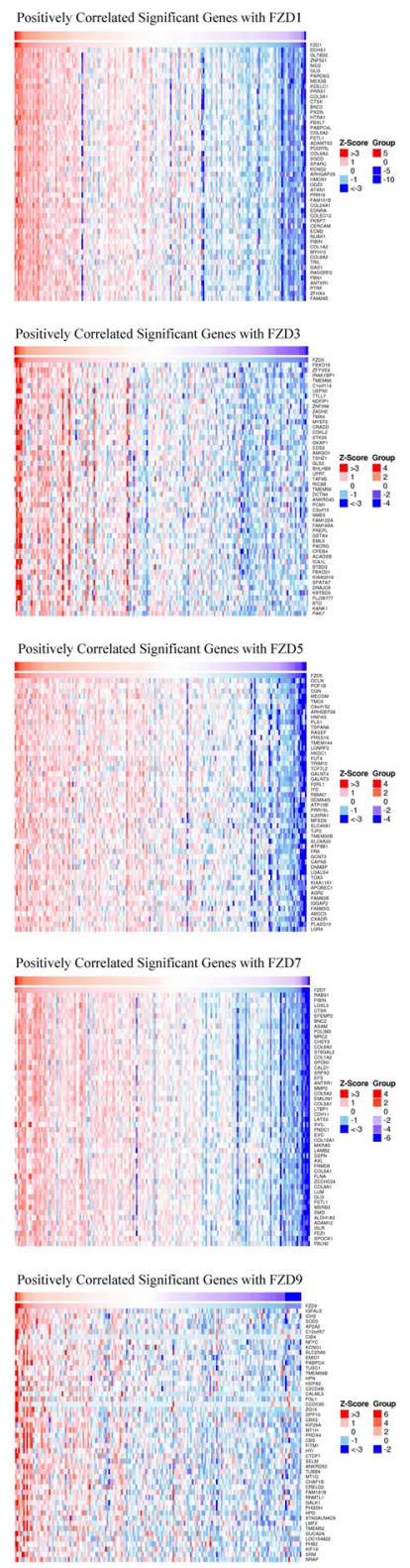
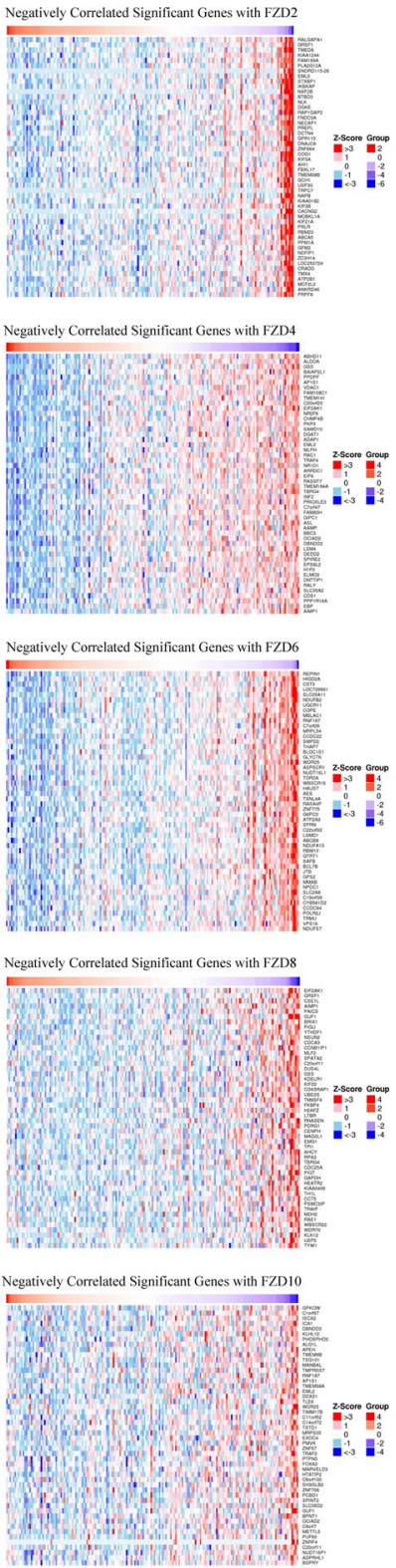
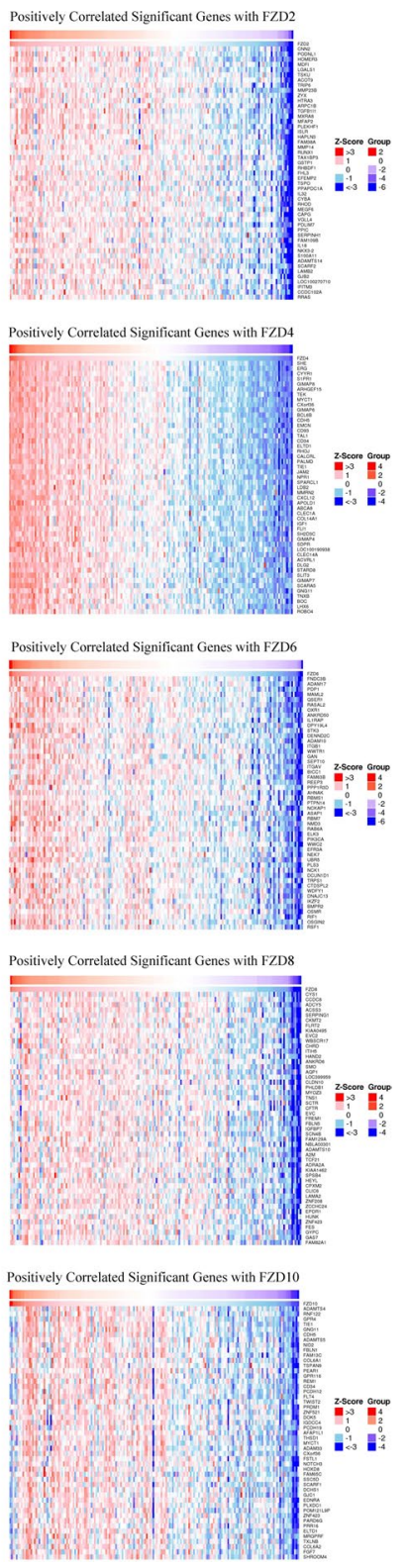

and induced the progression of epithelial-mesenchymal transition (EMT) [20]. But FZD2 showed no effect on the cell growth of endometrial cancer. In our study, PAAD patients with stage IV had the highest expression of FZD2 compared with stage I, II, and III, suggesting FZD2 might also participate in metastatic events of PAAD.

FZD6, a 7-transmem-brane domain receptor of Wnt pathway, was reported to play an essential role in digestive tract tumors. The combined expression of WNT11 and FZD6 could be predictive of a worse clinical outcome for patients with colorectal cancer [21]. NPTX2 promoted the proliferation and metastasis of colorectal cancer cells through interacting with FZD6 [22]. In gastric cancer, Wnt3A and FZD6 mediated trastuzumab resistance by activating $\mathrm{Wnt} / \beta$-catenin pathway [23]. Yan et al. [24] reported that FZD6 could inhibit proliferation and migration by activating non-canonical Wnt signaling pathway in gastric cancer. In contrast to Yan et al., FZD6 may act as a cancer-promoting gene in PAAD since FZD6 was closely related to poor clinical outcomes. Yang et al. [25] also showed that DLX6-AS1/miR-497-5p/FZD4/ 


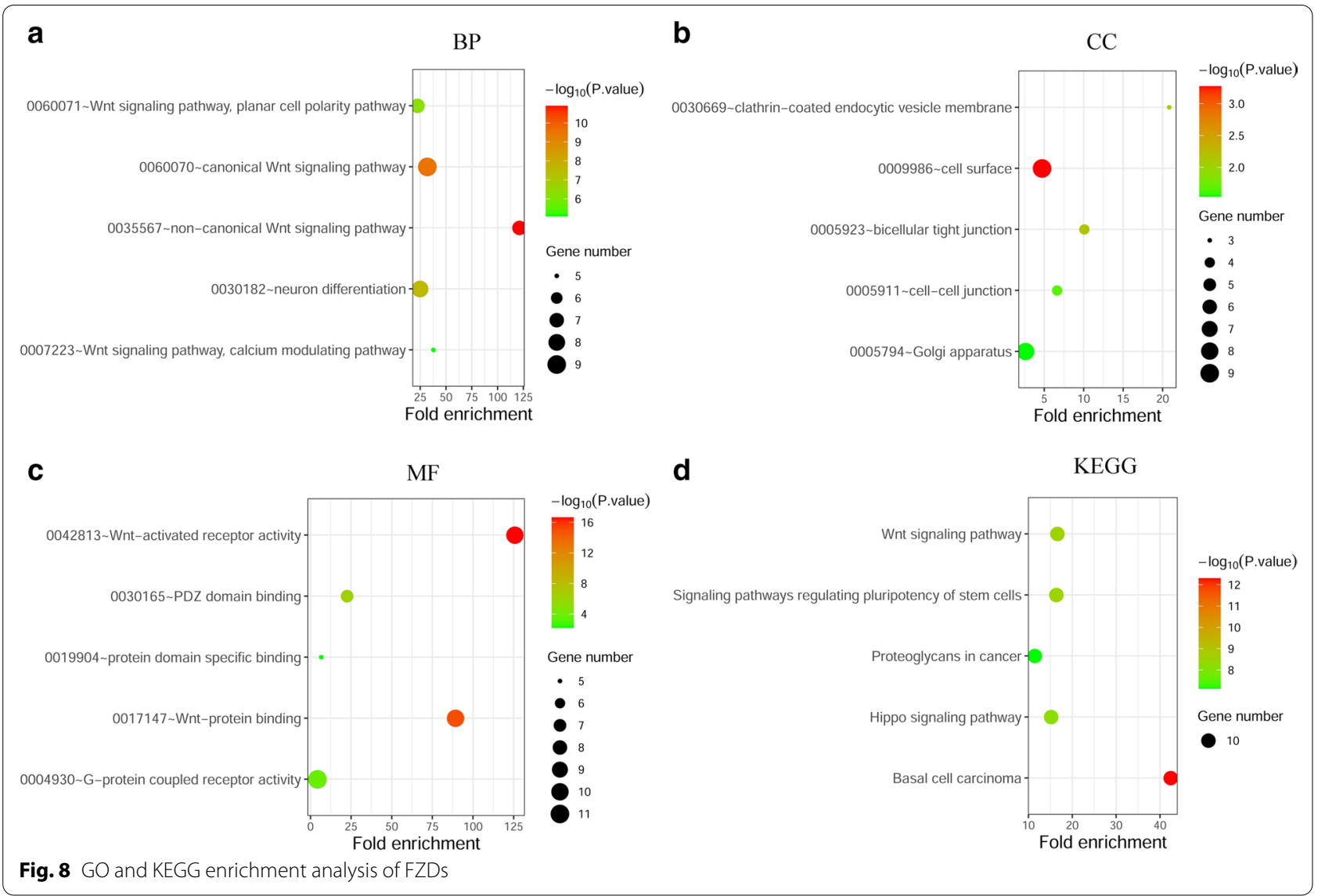

FZD6/Wnt/ $\beta$-catenin pathway promoted tumorigenesis of PAAD. Therefore FZD6 might function as a very promising therapeutic target in PAAD by blocking Wnt/ $\beta$-catenin pathway.

With respect to FZD7, the vast majority of studies focused on breast cancer, in particular triple-negative breast cancer (TNBC), for TNBC was unresponsive to classical endocrine therapy. FZD7 was thus considered as one of the important therapeutic targets in TNBC and showed an improved therapeutic efficacy as well [26-30]. However, little is known about the role of FZD7 in PAAD to date. We found that the expression level of FZD7 mRNA was increased in PAAD and could potentially be a therapeutic target.

Mounting evidences showed that FZD8 was involved in various malignant tumors including prostate cancer [31-33], lung cancer[34], breast cancer [35], and gastric cancer[36]. Yin et al. [37] suggested that FZD8 had an important role in resistance to treatment with cisplatin plus TRAIL in patients triple-negative breast cancer, thus making it a potential target for chemosensitization. Wang et al. [38] found that lung cancer cells would be more sensitive to Taxotere when the expression of FZD8 was down-regulated. Using RNF43-mutant PAAD cells,
Zachary et al. [16] discovered that anti-FZD5 and antiFZD8 antibodies could repress the cancer cell growth, providing support for chemotherapeutics development in PAAD. Consistent with these results, we demonstrated that FZD8 would result in a high recurrence rate and could become a potential therapeutic target in PAAD patients. In fact, an evaluation of efficacy of Vantictumab in PAAD had entered the phase I clinical trial, which exhibited specific binding to FZD1, 2, 5, 7, and 8 receptors [39]. The optimal dosage of Vantictumab are still under exploration.

There was no significant difference in expression levels of FZD3, 4, 5 and 9 between PAAD and normal tissues, whereas the prognosis of patients with high expression groups was significantly different from those with low expression groups. Currently, there is very little understanding of their exact functions in PAAD. For example, FZD3 was proved to be required for the development of embryonic pancreas in mice [40]. FZD4 may be involved in the regulation of $\beta$-cell functions in mouse [41]. More gene and clinical evidence are needed to explore their potential values.

There were a few limitations in the study. First, the study is short of the verification of biological or 


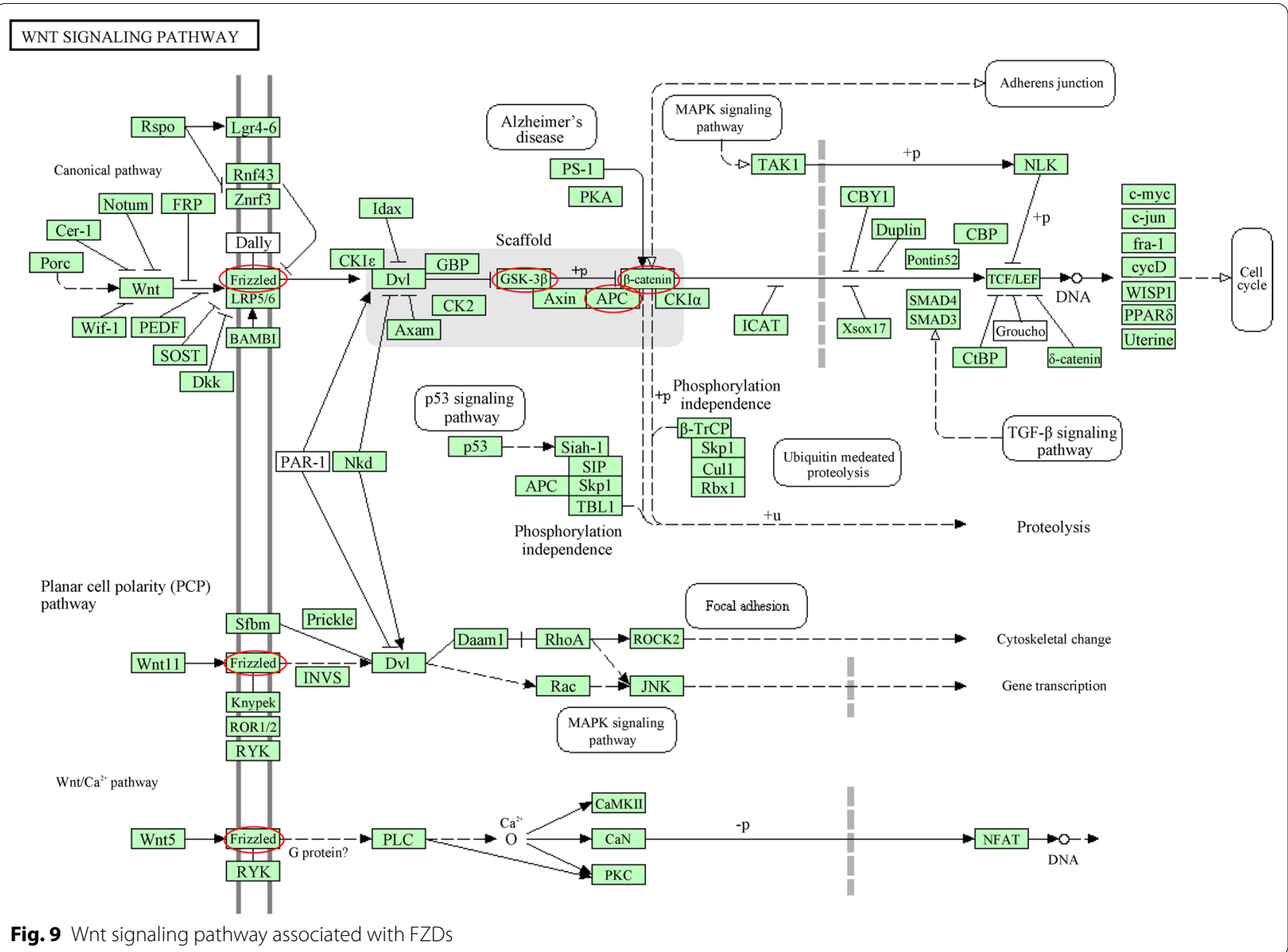

molecular experiments. Second, PAAD shows strong heterogeneity [42] while the mRNA expression levels from the TCGA database are the average mRNA expression levels of all cell types within the tumor. Single-cell sequencing is needed to further elaborate the role of FZDs in PAAD.

\section{Conclusion}

To sum up, our results strongly indicated a crucial role of the FZD family in PAAD. FZD3 and FZD6 could be potential prognostic and predictive markers, and FZD6 might also function as a potential therapeutic target in PAAD by blocking Wnt/ $\beta$-catenin pathway.

\section{Abbreviations}

PAAD: Pancreatic adenocarcinoma; HCC: Hepatocellular carcinoma; DLBCL: Diffuse large B-cell lymphoma; CRDs: Cystein-rich domains; FC: Fold change; GEPIA: Gene expression profiling interactive analysis; TCGA: The Cancer Genome Atlas; GTEx: Genotype-Tissue Expression project; ANOVA: Analysis of variance; HPA: Human protein atlas; OS: Overall survival; RFS: Recurrence free survival; HR: Hazard ratio; GO: Gene ontology; KEGG: Kyoto Encyclopedia of Genes and Genomes; BP: Biological processes; CC: Cellular component; MF:
Molecular function; EMT: Epithelial-mesenchymal transition; TNBC: Triplenegative breast cancer; $\mathrm{Cl}$ : Confidence interval.

\section{Acknowledgements}

None.

Authors' contributions

YL and YZ conceived the study, designed the experiments, collected data, and wrote the manuscript. ZL conducted the experiments and performed data analysis. All authors read and approved the final version of the paper.

\section{Funding}

This work was supported by Key Projects of Tianjin Health Industry (16KG108) and Tianjin Science and Technology Plan Project (19ZXDBSY00010).

\section{Availability of data and materials}

The datasets generated and analyzed during the current study are available from the corresponding author on reasonable request. The raw data could also be obtained from online databases including the TCGA, Oncomine, HPA, cBioPortal, and LinkedOmics without any restrictions.

\section{Ethics approval and consent to participate}

The study protocol was approved by the Ethics Committee of Tianjin First Central Hospital. All patients from the TCGA database gave written consent to participate in any scientific researches.

\section{Consent for publication}

Not applicable. 


\section{Competing interests}

The authors declare that they have no competing interests.

Received: 16 September 2020 Accepted: 3 February 2021 Published online: 22 February 2021

\section{References}

1. Wang X, Wang L, Mo Q, Dong Y, Wang G, Ji A. Changes of Th17/Treg cell and related cytokines in pancreatic cancer patients. Int J Clin Exp Pathol. 2015;8(5):5702-8

2. Siegel RL, Miller KD, Jemal A. Cancer statistics, 2020. CA Cancer J Clin. 2020;70(1):7-30.

3. Xu D, Allsop SA, Witherspoon SM, Snider JL, Yeh JJ, Fiordalisi JJ, White CD, Williams D, Cox AD, Baines AT. The oncogenic kinase Pim-1 is modulated by K-Ras signaling and mediates transformed growth and radioresistance in human pancreatic ductal adenocarcinoma cells. Carcinogenesis. 2011;32(4):488-95.

4. Sahu RP, Zhang R, Batra S, Shi Y, Srivastava SK. Benzyl isothiocyanatemediated generation of reactive oxygen species causes cell cycle arrest and induces apoptosis via activation of MAPK in human pancreatic cancer cells. Carcinogenesis. 2009;30(10):1744-53.

5. Ma C, Huang T, Ding YC, Yu W, Wang Q, Meng B, Luo SX. MicroRNA200c overexpression inhibits chemoresistance, invasion and colony formation of human pancreatic cancer stem cells. Int J Clin Exp Pathol. 2015;8(6):6533-9.

6. Park JK, Doseff Al, Schmittgen TD. MicroRNAs targeting caspase-3 and -7 in PANC-1 cells. Int J Mol Sci. 2018;19(4):1206.

7. Ghosh MC, Collins GD, Vandanmagsar B, Patel K, Brill M, Carter A, Lustig A, Becker KG, Wood WW III, Emeche CD, et al. Activation of Wnt5A signaling is required for $\mathrm{CXC}$ chemokine ligand 12-mediated T-cell migration. Blood. 2009;114(7):1366-73.

8. Arthofer E, Hot B, Petersen J, Strakova K, Jager S, Grundmann M, Kostenis E, Gutkind JS, Schulte G. WNT stimulation dissociates a frizzled 4 inactivestate complex with Galpha12/13. Mol Pharmacol. 2016;90(4):447-59.

9. Peng C, Xiao X, Kang B, He S, Li J. Serum secreted frizzled-related protein 5 levels differentially decrease in patients with hepatitis $B$ virusassociated chronic infection and hepatocellular carcinoma. Oncol Lett. 2014;8(3):1340-4.

10. McIntyre DC, Seay NW, Croce JC, McClay DR. Short-range Wnt5 signaling initiates specification of sea urchin posterior ectoderm. Development. 2013;140(24):4881-9.

11. Huang L, Luo EL, Xie J, Gan RH, Ding LC, Su BH, Zhao Y, Lin LS, Zheng DL, LU YG. FZD2 regulates cell proliferation and invasion in tongue squamous cell carcinoma. Int J Biol Sci. 2019;15(11):2330-9.

12. Chen L, Long Y, Han Z, Yuan Z, Liu W, Yang F, Li T, Shu L, Zhong Y. MicroRNA-101 inhibits cell migration and invasion in bladder cancer via targeting FZD4. Exp Ther Med. 2019;17(2):1476-85.

13. Hu S, Xu-Monette ZY, Balasubramanyam A, Manyam GC, Visco C, Tzankov A, Liu WM, Miranda RN, Zhang L, Montes-Moreno S, et al. CD30 expression defines a novel subgroup of diffuse large B-cell lymphoma with favorable prognosis and distinct gene expression signature: a report from the International DLBCL Rituximab-CHOP Consortium Program Study. Blood. 2013;121(14):2715-24.

14. Liu B, Cao G, Dong Z, Guo T. Effect of microRNA-27b on cisplatin chemotherapy sensitivity of oral squamous cell carcinoma via FZD7 signaling pathway. Oncol Lett. 2019;18(1):667-73.

15. Fakhar M, Najumuddin, Gul M, Rashid S. Antagonistic role of Klothoderived peptides dynamics in the pancreatic cancer treatment through obstructing WNT-1 and Frizzled binding. Biophys Chem. 2018;240:107-17.

16. Steinhart Z, Pavlovic Z, Chandrashekhar M, Hart T, Wang X, Zhang X, Robitaille M, Brown KR, Jaksani S, Overmeer R, et al. Genome-wide CRISPR screens reveal a Wnt-FZD5 signaling circuit as a druggable vulnerability of RNF43-mutant pancreatic tumors. Nat Med. 2017;23(1):60-8.

17. Yang L, Yang Z, Li D, Liu Z, Zou Q, Yuan Y, Xu H. Overexpression of FZD1 and CAIX are associated with invasion, metastasis, and poorprognosis of the pancreatic ductal adenocarcinoma. Pathol Oncol Res. 2018;24(4):899-906.
18. Garg B, Giri B, Majumder K, Dudeja V, Banerjee S, Saluja A. Modulation of post-translational modifications in beta-catenin and LRP6 inhibits Wnt signaling pathway in pancreatic cancer. Cancer Lett. 2017;388:64-72.

19. Tomizawa M, Shinozaki F, Motoyoshi Y, Sugiyama T, Yamamoto S, Ishige N. Gastric cancer cell proliferation is suppressed by frizzled-2 short hairpin RNA. Int J Oncol. 2015;46(3):1018-24.

20. Bian Y, Chang X, Liao Y, Wang J, Li Y, Wang K, Wan X. Promotion of epithelial-mesenchymal transition by Frizzled 2 is involved in the metastasis of endometrial cancer. Oncol Rep. 2016;36(2):803-10.

21. Gorrono-Etxebarria I, Aguirre U, Sanchez S, Gonzalez N, Escobar A, Zabalza I, Quintana JM, Vivanco MD, Waxman J, Kypta RM. Wnt-11 as a potential prognostic biomarker and therapeutic target in colorectal cancer. Cancers (Basel). 2019;11(7):908.

22. Xu C, Tian G, Jiang C, Xue H, Kuerbanjiang M, Sun L, Gu L, Zhou H, Liu $Y$, Zhang Z, et al. NPTX2 promotes colorectal cancer growth and liver metastasis by the activation of the canonical Wnt/beta-catenin pathway via FZD6. Cell Death Dis. 2019;10(3):217.

23. Liu W, Yuan J, Liu Z, Zhang J, Chang J. Label-free quantitative proteomics combined with biological validation reveals activation of Wnt/betacatenin pathway contributing to trastuzumab resistance in gastric cancer. Int J Mol Sci. 2018;19(7):1981.

24. Yan J, Liu T, Zhou X, Dang Y, Yin C, Zhang G. FZD6, targeted by miR-21, represses gastric cancer cell proliferation and migration via activating non-canonical wnt pathway. Am J Transl Res. 2016;8(5):2354-64.

25. Yang J, Ye Z, Mei D, Gu H, Zhang J. Long noncoding RNA DLX6-AS1 promotes tumorigenesis by modulating miR-497-5p/FZD4/FZD6/ Wnt/beta-catenin pathway in pancreatic cancer. Cancer Manag Res. 2019;11:4209-21.

26. Xie W, Zhang Y, He Y, Zhang K, Wan G, Huang Y, Zhou Z, Huang G, Wang J. A novel recombinant human Frizzled-7 protein exhibits anti-tumor activity against triple negative breast cancer via abating Wnt/beta-catenin pathway. Int J Biochem Cell Biol. 2018;103:45-55.

27. Arzi L, Riazi G, Sadeghizadeh M, Hoshyar R, Jafarzadeh N. A comparative study on anti-invasion, antimigration, and antiadhesion effects of the bioactive carotenoids of saffron on $4 \mathrm{~T} 1$ breast cancer cells through their effects on Wnt/beta-catenin pathway genes. DNA Cell Biol. 2018:37(8):697-707.

28. Jia XP, Meng LL, Fang JC, Wang HW, Chen J, Zhou J, Wang CN, Jiang WF. Aberrant expression of miR-142-3p and its target gene HMGA1 and FZD7 in breast cancer and its clinical significance. Clin Lab. 2018;64(6):915-21.

29. Zarei N, Fazeli M, Mohammadi M, Nejatollahi F. Cell growth inhibition and apoptosis in breast cancer cells induced by anti-FZD7 scFvs: involvement of bioinformatics-based design of novel epitopes. Breast Cancer Res Treat. 2018;169(3):427-36.

30. Riley RS, Day ES. Frizzled7 antibody-functionalized nanoshells enable multivalent binding for Wnt signaling inhibition in triple negative breast cancer cells. Small. 2017;13(26):1700544.

31. Wang Y, Singhal U, Qiao Y, Kasputis T, Chung JS, Zhao H, Chammaa F, Belardo JA, Roth TM, Zhang H, et al. Wnt Signaling Drives Prostate Cancer Bone Metastatic Tropism and Invasion. Transl Oncol. 2020;13(4):100747.

32. Chakravarthi B, Chandrashekar DS, Hodigere Balasubramanya SA, Robinson AD, Carskadon S, Rao U, Gordetsky J, Manne U, Netto GJ, Sudarshan S, et al. Wnt receptor Frizzled 8 is a target of ERG in prostate cancer. Prostate. 2018;78(16):1311-20.

33. Li Q, Ye L, Zhang X, Wang M, Lin C, Huang S, Guo W, Lai Y, Du H, Li J, et al. FZD8, a target of $\mathrm{p} 53$, promotes bone metastasis in prostate cancer by activating canonical Wnt/beta-catenin signaling. Cancer Lett. 2017:402:166-76.

34. Zhang G, He L, Chen J, Xu B, Mao Z. Ginsenoside Rh2 activates alphacatenin phosphorylation to inhibit lung cancer cell proliferation and invasion. Exp Ther Med. 2020;19(4):2913-22.

35. Park AY, Han MR, Park KH, Kim JS, Son GS, Lee HY, Chang YW, Park EK, Cha $\mathrm{SH}, \mathrm{Cho}$ Y, et al. Radiogenomic analysis of breast cancer by using B-mode and vascular US and RNA sequencing. Radiology. 2020;295(1):24-34.

36. Chen W, Liu Z, Mai W, Xiao Y, You X, Qin L. FZD8 indicates a poor prognosis and promotes gastric cancer invasion and metastasis via B-catenin signaling pathway. Ann Clin Lab Sci. 2020;50(1):13-23.

37. Yin S, Xu L, Bonfil RD, Banerjee S, Sarkar FH, Sethi S, Reddy KB. Tumorinitiating cells and FZD8 play a major role in drug resistance in triplenegative breast cancer. Mol Cancer Ther. 2013;12(4):491-8. 
38. Wang HQ, Xu ML, Ma J, Zhang Y, Xie CH. Frizzled-8 as a putative therapeutic target in human lung cancer. Biochem Biophys Res Commun. 2012;417(1):62-6.

39. Davis SL, Cardin DB, Shahda S, Lenz HJ, Dotan E, O'Neil BH, Kapoun AM, Stagg RJ, Berlin J, Messersmith WA, et al. A phase 1b dose escalation study of Wnt pathway inhibitor vantictumab in combination with nab-paclitaxel and gemcitabine in patients with previously untreated metastatic pancreatic cancer. Invest New Drugs. 2020;38(3):821-30.

40. Papadopoulou S, Edlund $\mathrm{H}$. Attenuated Wnt signaling perturbs pancreatic growth but not pancreatic function. Diabetes. 2005;54(10):2844-51.

41. Kurita Y, Ohki T, Soejima E, Yuan X, Kakino S, Wada N, Hashinaga T, Nakayama H, Tani J, Tajiri Y, et al. A high-fat/high-sucrose diet induces
WNT4 expression in mouse pancreatic beta-cells. Kurume Med J. 2019;65(2):55-62.

42. Tong $M$, Wang J, Zhang $H$, Xing $H$, Wang $Y$, Fang $Y$, Pan H, Li D. Efficacy and safety of gemcitabine plus anti-angiogenesis therapy for advanced pancreatic cancer: a systematic review and meta-analysis of clinical randomized phase III trials. J Cancer. 2019;10(4):968-78.

\section{Publisher's Note}

Springer Nature remains neutral with regard to jurisdictional claims in published maps and institutional affiliations.
Ready to submit your research? Choose BMC and benefit from:

- fast, convenient online submission

- thorough peer review by experienced researchers in your field

- rapid publication on acceptance

- support for research data, including large and complex data types

- gold Open Access which fosters wider collaboration and increased citations

- maximum visibility for your research: over 100M website views per year

At BMC, research is always in progress.

Learn more biomedcentral.com/submissions 